\title{
Controlling dynamical behavior of a semiconductor laser with external optical feedback
}

\author{
Yun Liu \\ Graduate School of Electronic Science and Technology, Shizuoka University, Johoku 3-5-1, Hamamatsu 432, Japan \\ Noriyuki Kikuchi and Junji Ohtsubo \\ Faculty of Engineering, Shizuoka University, Johoku 3-5-1, Hamamatsu 432, Japan
}

(Received 15 November 1994)

\begin{abstract}
We describe controlling chaos in a laser diode with an external cavity through a sinusoidal modulation to the injection current. The modulation is optimized by analytically determining the modulation frequency from the mode analysis of the dynamical model. The numerical results show that chaos can be successfully stabilized to various limit cycles with very small modulation amplitudes.
\end{abstract}

PACS number(s): 05.45. $+\mathrm{b}, 42.50 . \mathrm{Ne}, 42.55 . \mathrm{Px}$

Nonlinear dynamics of laser diodes with external optical feedback has received much attention in recent years from both theoretical and experimental aspects. Since a laser diode with optical feedback provides a very excellent all-optical chaos model, a large number of studies have been performed on such systems to investigate both static and dynamical behaviors such as mode hops, coherence collapse, and bifurcations to chaos [1]. The study of nonlinear dynamics in laser diodes has also been of great importance to the applications of laser diodes to optical communications and optical data recording systems. In most practical environments, some kind of optical feedback to the laser diodes is inevitably induced and, sometimes, such optical feedback becomes very bothersome because a feedback level as small as $0.1 \%$ may cause intensity noise enhancement that dramatically deteriorates the performance. Therefore some methods have been proposed to avoid such feedback induced effects. One of the methods that is currently used in optical data recording systems is known as a high-frequency injection (HFI) technique, which modulates the laser diode injection current with frequencies much higher than the data rate $[2,3]$. The experimental results show that the relative intensity noise (RIN) enhancement does not occur if the modulation frequency is appropriately chosen and if the modulation amplitude is large enough. However, the optimum value of the modulation frequency has to be determined empirically and, up to now, only a few numerical analyses have been performed to understand the mechanism of such HFI techniques [3].

In this paper, we try to give a solution to the frequency optimization in the HFI technique from a chaos controlling point of view. We consider that the feedback induced deterministic instabilities are responsible for the intensity noise enhancement. Hence one can realize suppression or control of the feedback induced intensity noise enhancement provided the feedback induced chaos is effectively stabilized. Therefore the optimization of the modulation frequency is converted to a problem of how to select the frequency by which chaos can be most effectively stabilized. In the following, we first present the numerical calculations of the rate equation model of the laser diode with optical feedback and investigate the bifurcation routes under different parameter conditions. Then the linear stability analysis is performed which yields the mode distributions. Based on the mode distributions, one can choose to strengthen a certain mode by modulating the laser diode injection current with a sinusoidal signal whose frequency is equal to the corresponding mode. As a result, the original chaos is stabilized to a limit cycle. The algorithm is numerically verified by a computer simulation. Possible applications of the technique to the optimization of the modulation frequency in the HFI are also described.

The schematic of the laser diode with external cavity is shown in Fig. 1. The light source considered in this paper is a channeled substrate planar (CSP) $\mathrm{Al}_{x} \mathrm{Ga}_{1-x} \mathrm{As}$ semiconductor laser with the cavity length $l$ and the refractive index $\eta$. The internal amplitude reflectivities of the front and rear facets of the laser diode are $r_{1}$ and $r_{2}$, respectively. In this paper, we consider the case $r_{1}=r_{2}$. The light beam is reflected by an external mirror located at a distance $L$ from the front facet of the cavity. The fraction of the electric amplitude of the output light coupled into the cavity is denoted as $r_{3}$, which includes the amplitude reflectivity of the external mirror, the propagating losses in the external optical path, and the coupling efficiency.

We choose the carrier density $N(t)$ and the complex electric field $E(t)$ as the dynamic variables of the system. $N(t)$ is the average carrier density in the active region, $E(t)$ is the electric field at the front facet and can be divided into its real amplitude and phase factors as $E(t)=E_{0}(t) \exp \left\{j\left[\left(\omega_{0} t\right.\right.\right.$ $+\phi(t)]\}$, where $\omega_{0}$ is the angular frequency of the solitary laser. For the weak to moderate feedback level, we have the following rate equations:

$$
\begin{aligned}
\frac{d E_{0}(t)}{d t}= & \frac{1}{2}\left\{G_{N}\left[N(t)-N_{0}\right]-\frac{1}{\tau_{p}}\right\} E_{0}(t) \\
& +\frac{\kappa}{\tau_{\text {in }}} E_{0}(t-\tau) \cos [\Delta(t)]+F_{I}(t),
\end{aligned}
$$

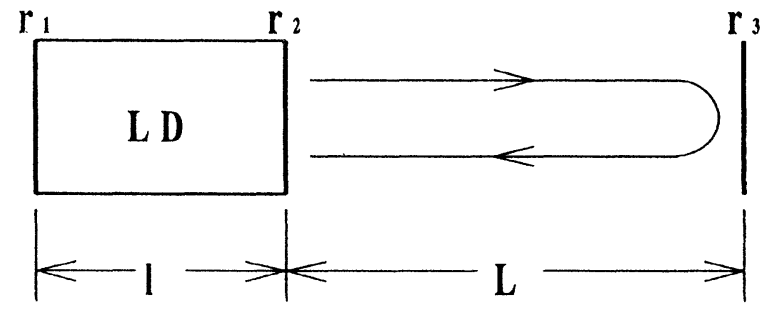

FIG. 1. Schematic of the laser diode with external optical feedback. 


$$
\begin{array}{r}
\frac{d \Phi(t)}{d t}=\frac{\alpha}{2} G_{N}\left[N(t)-N_{t h}\right]-\frac{\kappa E_{0}(t-\tau)}{\tau_{i n} E_{0}(t)} \sin [\Delta(t)]+F_{\phi}(t), \\
\frac{d N(t)}{d t}=J-\frac{N(t)}{\tau_{s}}-G_{N}\left[N(t)-N_{0}\right]\left|E_{0}(t)\right|^{2}+F_{N}(t),
\end{array}
$$

with

$$
\Delta(t)=\omega_{0} \tau+\phi(t)-\phi(t-\tau)
$$

Here, $G_{N}$ is the model gain coefficient, $N_{0}$ is the carrier density at the transparency, $N_{t h}$ is the threshold carrier density for the solitary laser, $\kappa=\left(1-r_{2}^{2}\right) r_{3} / r_{2}$ is the feedback coefficient, and $F_{I}, F_{N}$, and $F_{\phi}$ are Langevin noise terms. Also, $\tau_{p}$ is the photon lifetime, $\tau_{i n}=2 \eta l / c$ is the optical round trip time in the laser diode cavity, $\tau_{s}$ is the carrier lifetime, $\tau=2 L / c$ is the external cavity round trip time, $\alpha$ is the linewidth enhancement factor, and $J$ is the injection current density. We neglect the small contribution from nonlinear gain suppression and spontaneous emission. Some typical numerical values for the various parameters, as appropriate for a 780-nm $\mathrm{Al}_{x} \mathrm{Ga}_{1-x}$ As semiconductor laser likely to be used in an optical recording system, are given in Table I [1-3].

The dynamics of the laser diode with external cavity can be generalized as a simple delay-differential model like

$$
d \mathbf{X}(t) / d t=\mathbf{F}(\mathbf{X}(t), \mathbf{X}(t-\tau) ; \mu),
$$

where $\mathbf{X}(t)$ is a vector variable, $\mathbf{F}$ is a nonlinear vector field, $\tau$ is the delay time introduced by the external feedback, $\mu$ is a system control parameter and may correspond to the injection current density $J$ or the effective external reflectivity $r_{3}$. In view of the dynamics of the delay-differential system, two sets of bifurcations can generally be observed in such a system. One is obtained by varying the system control parameter; the other is obtained by varying the delay time, i.e., the external cavity length in our case.

We have numerically calculated Eqs. (1)-(3) by employing a fourth order Runge-Kutta algorithm and verified various phase transitions among steady, periodic, quasiperiodic, and chaotic states. Here we only give a brief description about the bifurcation routes observed in our numerical investigations. The first one is the result via the variations of the effective external reflectivity $r_{3}$. As $r_{3}$ is increased from $1 \%$ to $2 \%$, we most often observed a continuous bifurcation from steady states to periodic states, then quasiperiodic states, and finally chaos. An incomplete period-doubling bifurcation also occurs for certain values of $r_{3}$, which leads to the subharmonic periodic solutions. Quasiperiodic solutions emerge from the fundamental periodic solution and the subharmonic periodic solutions or even the other limit cycles. Bifurcations upon the variation of the injection current become more complicated since such variation has also dramatically changed the steady state of the system. The system also evolves into chaos by varying the external cavity length only. In the calculations, we vary the external cavity length from $15 \mathrm{~cm}$ to $30 \mathrm{~cm}$; this corresponds to the change of the delay time from $1 \mathrm{~ns}$ to $2 \mathrm{~ns}$. It results in periodic solutions, subharmonic solutions, and chaos. Such a phenomenon is one of the generic properties of the delay-differential system: the output evolves into instability with the increase of the delay time. Two examples of bifurcations are plotted in Fig. 2 with
Figs. 2(a), 2(b), and 2(e) corresponding to the variation of $r_{3}$ and Figs. 2(c), 2(d), and 2(e) corresponding to the variation of $L$.

Before describing the control of chaos, we perform the stability analysis of the dynamical model and get the mode distributions. The steady-state solutions to $E_{0}, \phi$, and $N$ are obtained by substituting $E_{0}(t)=E_{s}, \quad N(t)=N_{s}$, and $\phi(t)=\left(\omega_{s}-\omega_{0}\right) t$ in Eqs. (1) $-(3)$,

$$
\begin{gathered}
\omega_{s}-\omega_{0}=-\left(\kappa / \tau_{i n}\right)\left[\alpha \cos \left(\omega_{s} \tau\right)+\sin \left(\omega_{s} \tau\right)\right], \\
G_{N}\left(N_{s}-N_{t h}\right)=-\left(2 \kappa / \tau_{i n}\right) \cos \left(\omega_{s} \tau\right), \\
G_{N}\left(N_{s}-N_{0}\right) E_{s}^{2}=J-\left(N_{s} / \tau_{s}\right) .
\end{gathered}
$$

In general, there exist a number of solutions of $\omega_{s}$ even for a fixed set of parameters. Among them we choose the one that results in the maximum gain as a steady-state solution of the angular frequency. The steady-state carrier density and field amplitude are uniquely determined from Eqs. (7) and (8) for a specific value of $\omega_{s} \tau$.

The stability properties of a particular steady-state solution are usually investigated by performing the linear expansion around that solution and by analyzing the stability to a small perturbation from the initial steady-state solution $[1,4,5]$. To this end, we adopt the following approximation: $x(t)=x_{s}+\delta x \exp (\gamma t)\left(x=E_{0}, N, \phi\right)$ and substitute into Eqs. (6)-(8). After some algebra, one gets a transcendental equation for $\gamma$

$$
\begin{aligned}
& \gamma^{3}+\left[\left(1 / \tau_{R}\right)+\right.\left.\left(2 \kappa A / \tau_{i n}\right) \cos \left(\omega_{s} \tau\right)\right] \gamma^{2}+\omega_{R}^{2} \gamma+\left(\kappa^{2} A^{2} / \tau_{R} \tau_{i n}^{2}\right) \\
&+\left(\kappa A / \tau_{i n}\right) \omega_{R}^{2}\left[\cos \left(\omega_{s} \tau\right)-\alpha \sin \left(\omega_{s} \tau\right)\right]=0,
\end{aligned}
$$

with $A=1-\exp (-\gamma \tau), \quad \tau_{R}^{-1}=\tau_{s}^{-1}+G_{N} E_{s}^{2}, \quad \omega_{R}^{2}=G_{N} E_{s}^{2}$ / $\tau_{p}$. Here, we have employed the relations $\omega_{R}^{2} \gg \kappa^{2} / \tau_{i n}^{2}$ and $\omega_{R}^{2} \gg \kappa / \tau_{R} \tau_{i n}$ which hold for a small or moderate feedback level. The above equation is called the characteristic equation or the eigenvalue equation of the model. Each solution, which is also called the linear mode, predicts how the small perturbation to the initial steady state evolves with respect to time development. The real part of the mode dominates the mode's stability and the imaginary part determines its angular oscillation frequency. As Eq. (9) is a transcendental equation, it can only be solved numerically. In general, it admits an infinite number of solutions. Although the dynamical output of the system is merely a nonlinear coupling of such linear modes and the system does not explicitly exhibit periodic oscillations corresponding to a particular mode, such linear modes, especially those near the stability criterion, do represent some intrinsic resonant frequencies of the system [4,5]. In our previous work, we have successfully exploited this knowledge in explaining dynamical phenomena, controlling chaos, and accessing high-mode oscillations of periodic

TABLE I. Some parameter values for laser diode used in the numerical simulations.

\begin{tabular}{lcc}
\hline \hline Symbol & Parameter & Value \\
\hline$G_{N}$ & gain coefficient & $8.4 \times 10^{-13} \mathrm{~m}^{3} \mathrm{~s}^{-1}$ \\
$\alpha$ & linewidth enhancement factor & 3 \\
$r_{1}, r_{2}$ & facet reflectivity & 0.556 \\
$N_{t h}$ & carrier density at threshold & $2.018 \times 10^{24} \mathrm{~m}^{-3}$ \\
$N_{0}$ & carrier density at transparency & $1.400 \times 10^{24} \mathrm{~m}^{-3}$ \\
$\tau_{s}$ & lifetime of carrier & $2.04 \mathrm{~ns}^{-1.927 \mathrm{ps}}$ \\
$\tau_{p}$ & lifetime of photon & $1.927 \mathrm{ps}$ \\
$\tau_{\text {in }}$ & round trip time in laser cavity & \\
\hline
\end{tabular}



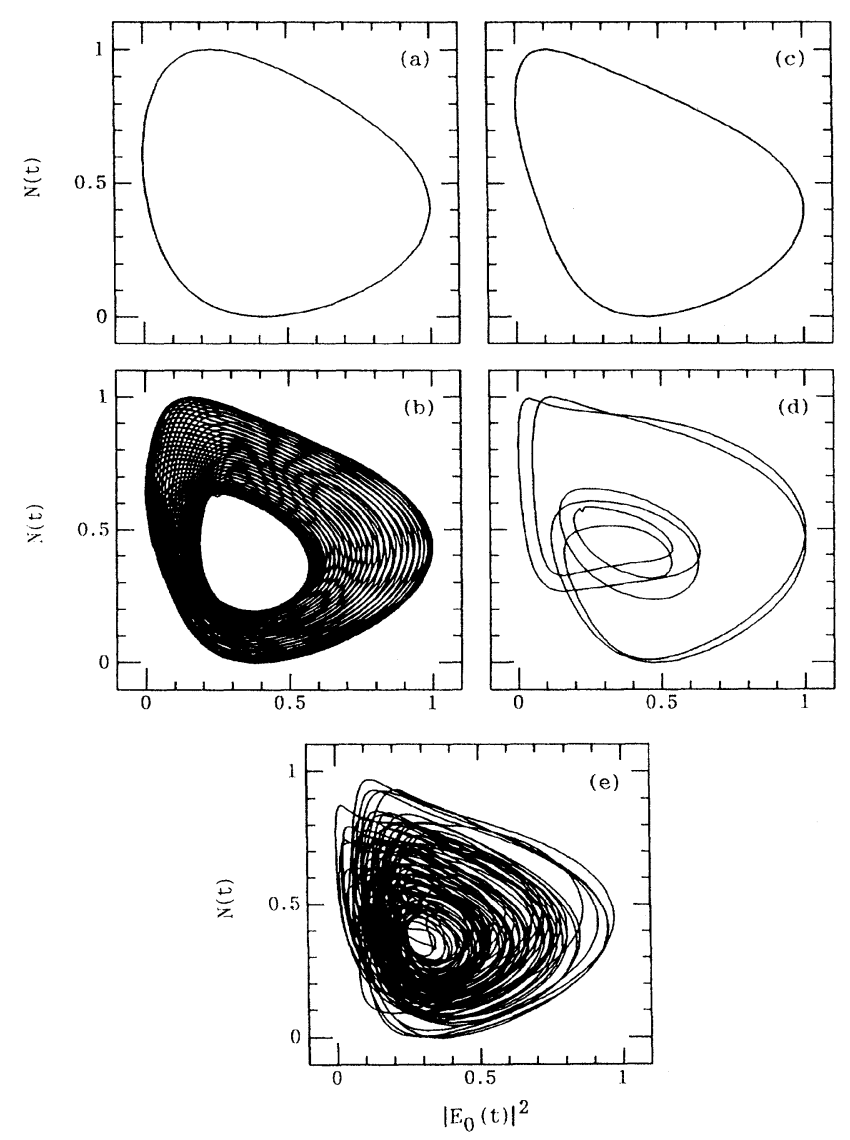

FIG. 2. Bifurcation route via $r_{3}$ and $L . J=1.1 J_{t h}$. (a) $r_{3}=1.1 \%$, $L=30 \mathrm{~cm}$, (b) $r_{3}=1.3 \%, L=30 \mathrm{~cm}$, (c) $r_{3}=1.5 \%, L=18 \mathrm{~cm}$, (d) $r_{3}=1.5 \%, L=24 \mathrm{~cm}$, and (e) $r_{3}=1.5 \%, L=30 \mathrm{~cm}$.

cycles in a delay-differential system [5]. The key point in stabilizing chaos with the sinusoidal modulation to the injection current is to assign the modulation frequency based on the above mode distributions. The algorithm can be written as

$$
J=J_{b}[1.0+\xi \sin (2 \pi \nu t)],
$$

where $J_{b}$ is the bias injection current density, $\xi$ is the modulation amplitude, and $\nu$ is the modulation frequency.

In Fig. 3, we show mode distributions for two typical chaotic output cases. In the figure, only modes which have lowest frequencies and whose real parts are very close to the stability criterion are plotted. It should be noted that the average separation between adjacent modes on the frequency axis is almost the same as the inverse of the delay time, which is in good agreement with the usual one-dimensional delay-differential model $[4,5]$.

The stabilization has been performed by modulating the injection current of the laser diode with frequencies equal to the imaginary parts of those modes indicated with arrows. Figure 4 shows the results of the stabilization for $J=1.1 J_{t h}, r_{3}=1.5 \%$, and $L=25.5 \mathrm{~cm}$, where $J_{t h}=N_{t h} / \tau_{s}$ is the current density at the threshold. The square modulus of the field amplitude $\left|E_{0}\right|^{2}$ is normalized to the light power emitted from the front facet. Figures 4(a) and 4(c) are the time series and the corresponding phase portrait for a chaotic output without the modulation, respectively, and Figs. 4(b)

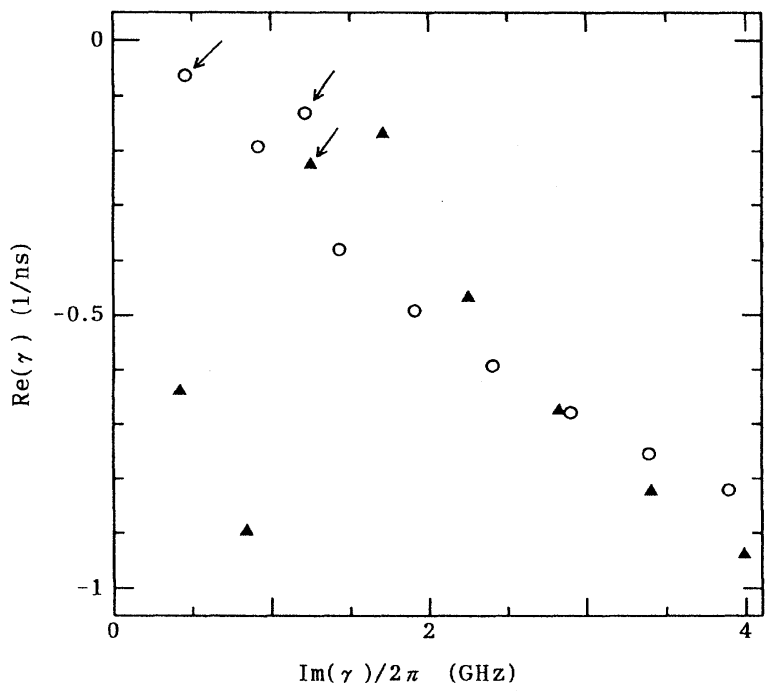

FIG. 3. Mode distributions for two typical chaotic outputs. Circles are for $J=1.07 J_{t h}, r_{3}=1.6 \%$, and $L=30 \mathrm{~cm}$, and solid triangles for $J=1.1 J_{t h}, r_{3}=1.5 \%$, and $L=25.5 \mathrm{~cm}$.

and 4(d) represent those after the modulation. The modulation frequency is chosen to be the same value of the largest stable mode shown in Fig. 2. Clearly the chaotic output is reduced to a limit cycle under such modulation frequency, though the modulation amplitude is as small as $2.1 \%$. Note that the amplitude of the periodic oscillations is about twice that of the original chaotic oscillations. This implies that the system is in a resonant state with respect to the frequency of the linear mode. Another example of the stabilization is shown in Fig. 5 for $J=1.07 J_{t h}, r_{3}=1.6 \%$, and $L=30 \mathrm{~cm}$. Here the largest two stable modes in Fig. 3 were chosen to modulate the injection current of the laser. One finds that the original chaotic output [Fig. 5(a)] is stabilized to two different limit cycles. The corresponding control conditions are $\nu=1.214 \mathrm{GHz}, \xi=2 \%$ for $5(\mathrm{~b})$ and $\nu=0.455 \mathrm{GHz}$, $\xi=2.5 \%$ for $5(\mathrm{c})$.

We have examined the robustness of the control to the small variations of both the modulation frequency and the amplitude. The results show that the modulation frequency has a variation range of about $100 \mathrm{MHz}$ and the modulation amplitude is variable within a range of $10 \%$ to even $20 \%$ of its ambient value. To make the problem simple, we did not include the noise terms in the above calculations. It should be noted that the control remains successful even if Langevin noise terms to some reasonable extent are included. The RIN level for both cases (with or without the modulation) has been calculated and the reduction of the RIN level with the modulation has been verified. The details will be published elsewhere.

Chaos control has been performed in various configurations [5-14]. We have noticed that chaos control through direct periodic modulation or parametric perturbation has been studied in different numerical and experimental systems [8-14]. However, there has been a lack of effective means so far for determining the modulation frequency which appeared to be a key factor in performing the control. On the other hand, with the control algorithm we proposed here, the modulation frequency can easily be evaluated from the known parameter values. As discussed in our previous 

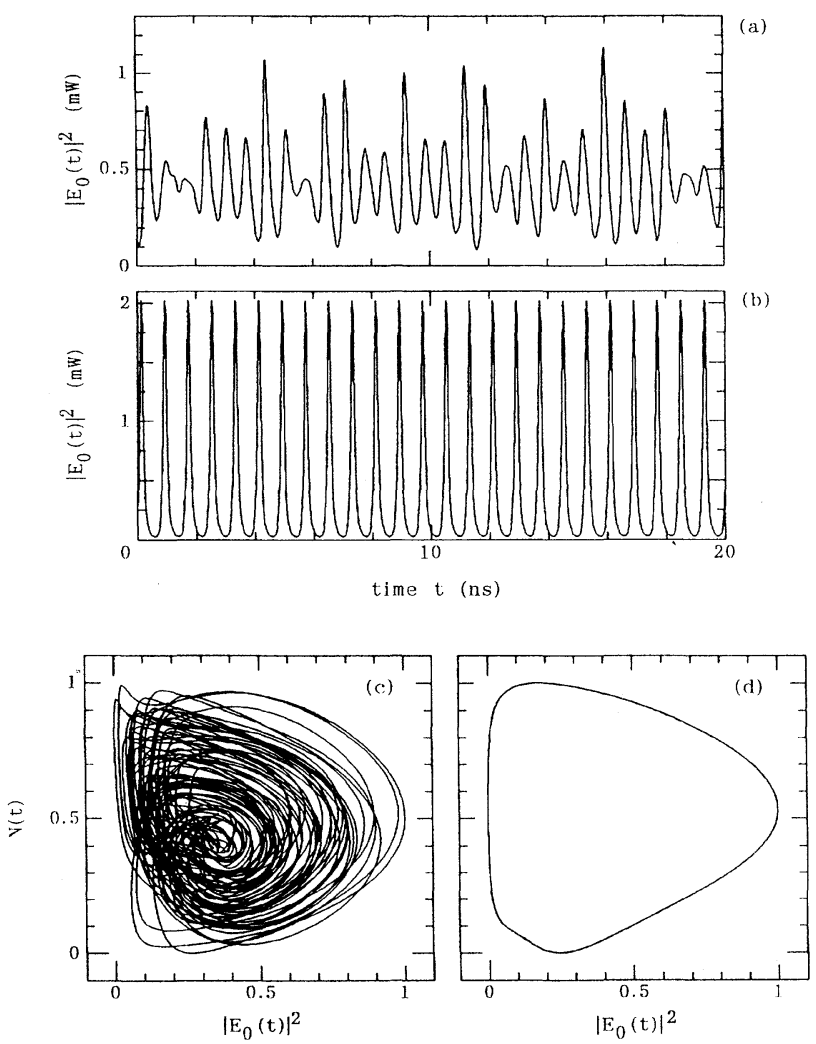

FIG. 4. Results of the control for $J=1.1 J_{t h}, r_{3}=1.5 \%$, and $L=25.5$ $\mathrm{cm}$. (a) Time series of chaotic output in the absence of the modulation, (b) periodic oscillations with the modulation of $\xi=0.021$ and $\nu=1.251 \mathrm{GHz}$. (c),(d) Phase potentials for (a) and (b), respectively. Note that the modulation frequency is equal to the imaginary part of the mode shown in Fig. 3.

works, the linear mode obtained from the stability analysis represents the resonant feature of the system [5]. The mode distribution in Fig. 3 clearly indicates an important feature that the modulation frequency might be the harmonics of a fundamental one. A similar feature has also been recognized in various experiments $[10,11]$. Therefore the control method is very promising to apply to noise reduction in semiconductor lasers through the HFI technique, where a theoretical optimization of the modulation frequency is strongly required.

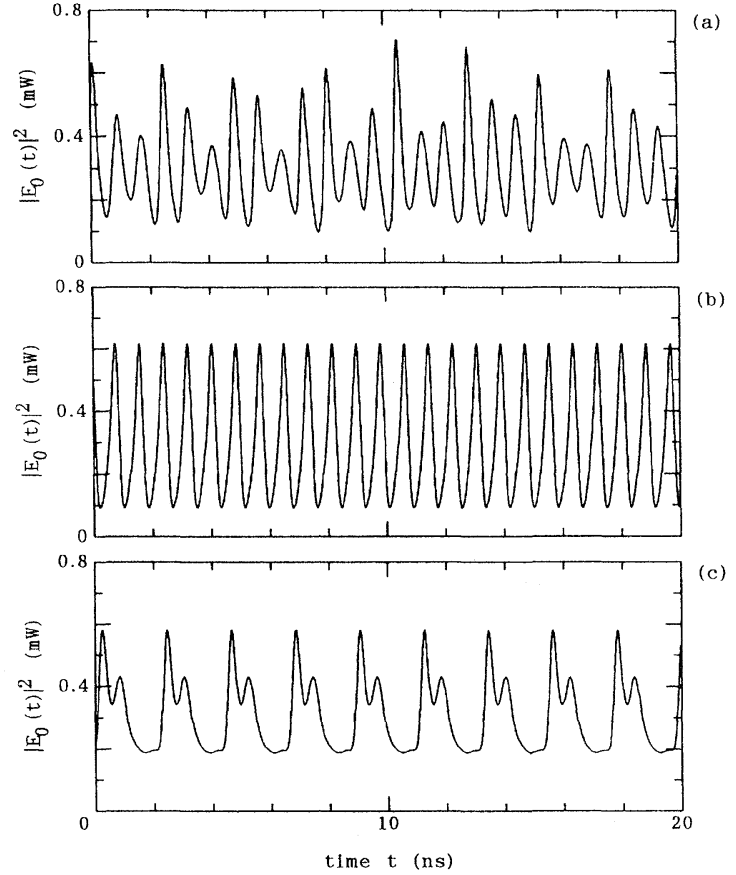

FIG. 5. Results of the control for $J=1.07 J_{t h}, r_{3}=1.6 \%$, and $L=30 \mathrm{~cm}$. (a) Chaotic output in the absence of the modulation, (b) periodic oscillation at $\xi=0.02$ and $\nu=1.214 \mathrm{GHz}$, and (c) periodic oscillation at $\xi=0.025$ and $\nu=0.455 \mathrm{GHz}$. Note the modulation frequencies are equal to the imaginary parts of the mode shown in Fig. 3.

In summary, we have conducted chaos control in semiconductor lasers with external optical feedback by superposing a high-frequency modulation on the injection current of the laser. The main advantage of the control algorithm is that the modulation frequencies can be analytically optimized from the mode analysis of the dynamical model of the system. It has been demonstrated numerically that chaos can be stabilized to different limit cycles under very weak modulations. The robustness of the control to the variations of the control parameters has been examined.

We gratefully acknowledge useful discussions of this work with R. Roy, E. R. Hunt, and G. H. M. van Tartwijk. This work was supported by The Mazda Foundation's Research Grant.
[1] For example, B. Tromborg et al., IEEE J. Quantum Electron. QE20, 1023 (1984); J. Mørk et al, ibid. QE28, 93 (1992); H. Li et al., ibid. QE29, 2421 (1993); H. Kakiuchida and J. Ohtsubo, ibid. QE30, 2087 (1994).

[2] T. Kanada, Trans. IEICE Jpn. 68, 180 (1985).

[3] M. Yamada and T. Higashi, IEEE J. Quantum Electron. QE27, 380 (1991); G. R. Gray et al., Opt. Eng. 32, 739 (1993).

[4] J. Y. Gao et al., Phys. Rev. A 30, 901 (1984); R. Vallée and C. Delisle, ibid. 34, 309 (1986).

[5] Y. Liu and J. Ohtsubo, Phys. Rev. A 47, 4392 (1993); Y. Liu et al., Opt. Commun. 105, 193 (1994); Y. Liu and J. Ohtsubo, Opt. Lett. 19, 448 (1994); Opt. Rev. 1, 91 (1994).

[6] E. Ott et al., Phys. Rev. Lett. 64, 1196 (1990).
[7] E. R. Hunt, Phys. Rev. Lett. 67, 1953 (1991); R. Roy et al., ibid. 68, 1259 (1992).

[8] Y. Braiman and I. Goldhirsch, Phys. Rev. Lett. 66, 2545 (1991).

[9] R. Chacón and J. D. Bejarano, Phys. Rev. Lett. 71, 3103 (1993).

[10] R. Lima and M. Pettini, Phys. Rev. A 41, 726 (1990); L. Fronzoni et al., ibid. 43, 6483 (1991).

[11] A. Azevedo and S. M. Rezende, Phys. Rev. Lett. 66, 1342 (1991).

[12] R. Meucci et al., Phys. Rev. E 49, R2528 (1994).

[13] Y. Liu and J. R. Rios Leite, Phys. Lett. A 185, 35 (1994).

[14] N. Watanabe and K. Karaki, Opt. Lett. (to be published). 